\title{
A phase 2 study of combined chemo-immunotherapy with cisplatin-pembrolizumab and radiation for unresectable vulvar squamous cell carcinoma
}

\author{
Oladapo Yeku ${ }^{1 *} \mathbb{B}$, Andrea L. Russo ${ }^{2}$, Hang Lee ${ }^{3}$ and David Spriggs ${ }^{1}$
}

\begin{abstract}
Background: Unresectable or metastatic vulvar cancer has relatively poor outcomes despite chemotherapy-sensitized radiation therapy and combination cytotoxic therapy. Despite the virus-associated and immunogenic nature of this disease, novel immunotherapy options that exploit this advantage are currently lacking. Platinum agents such as cisplatin have been shown to prime dendritic cells for T-cell costimulation, promote downregulation of inhibitory checkpoint molecules, and sensitize tumor cells to cytotoxic T-cell killing. Radiation therapy has also been shown to promote immunogenetic cell death as monotherapy and in combination with cisplatin. In combination with pembrolizumab, cisplatin-sensitized radiation is hypothesized to increase overall response rates and recurrence-free survival in patients with vulvar cancer, via induction of an anti-tumor inflammatory response.

Methods: We propose a single-arm phase II clinical trial of pembrolizumab combined with cisplatin-sensitized radiation therapy for women with unresectable, locally advanced, or metastatic vulvar cancer. The first three patients with locally advanced or unresectable disease will receive cycle 1 of pembrolizumab followed by a break and resumption of pembrolizumab at cycle 4 and as part of a safety cohort. All other patients, including the fourth patient with locally advanced/unresectable disease, will receive weekly cisplatin and pembrolizumab every 3 weeks, concurrently with daily radiation therapy. Following the completion of Cis-RT, patients will continue pembrolizumab maintenance for a total of 12 cycles. Archived tissue will be used for HPV status, MSI status, PD-L1, and TIL stratification post hoc. Imaging will be performed at baseline and every 3 cycles (21-day cycles) as per standard-of-care. Laboratory analysis will occur on the first day of each cycle.

Discussion: The combination of cisplatin-sensitized radiation and immune checkpoint blockade has not been evaluated in the upfront setting for vulvar cancer. In this rare malignancy, there are limited interventional clinical trials. This trial is designed to be as accessible as possible by allowing patients to receive cisplatin and radiation locally according to accepted standard-of-care while receiving pembrolizumab and adverse event monitoring at a centralized site. A robust suite of translational correlative studies has also been built into the trial to evaluate tumor-directed immune activation.
\end{abstract}

\footnotetext{
*Correspondence: Oyeku@mgh.harvard.edu

${ }^{1}$ Division of Hematology-Oncology, Department of Medicine, Harvard

Medical School, Massachusetts General Hospital, Boston, USA

Full list of author information is available at the end of the article
}

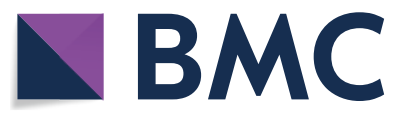

(c) The Author(s) 2020. This article is licensed under a Creative Commons Attribution 4.0 International License, which permits use, sharing, adaptation, distribution and reproduction in any medium or format, as long as you give appropriate credit to the original author(s) and the source, provide a link to the Creative Commons licence, and indicate if changes were made. The images or other third party material in this article are included in the article's Creative Commons licence, unless indicated otherwise in a credit line to the material. If material is not included in the article's Creative Commons licence and your intended use is not permitted by statutory regulation or exceeds the permitted use, you will need to obtain permission directly from the copyright holder. To view a copy of this licence, visit http://creativecommons.org/licenses/by/4.0/. The Creative Commons Public Domain Dedication waiver (http://creativecommons.org/publicdomain/zero/1.0/) applies to the data made available in this article, unless otherwise stated in a credit line to the data. 
Trial registration NCT04430699

Keywords: Vulvar cancer, Pembrolizumab, Chemoradiation, Immunotherapy, Chemoimmunotherapy

\section{Background}

Vulvar cancer represents $4 \%$ of gynecologic malignancies in the United States with an estimated incidence of 6190 women diagnosed in 2018 . Of these, $20 \%$ are expected to die from disease [1]. The primary treatment for locally advanced or recurrent metastatic disease is highly-individualized, and typically involves some combination of surgery, radiation therapy (RT) with or without radiosensitization and platinum-based doublet chemotherapy [2]. Patients with locally advanced or metastatic disease at presentation have a 5-year survival of 53\% and 19\% respectively [3]. Patients with recurrent metastatic disease have survival rates between 14 and 15\% [2]. Furthermore, no standard-of-care exists for patients who recur or relapse after primary therapy. Platinum-based combination chemotherapy for advanced or recurrent metastatic vulvar cancer has an overall response rate (ORR) of $40 \%$ [4], while single-agent paclitaxel has a response rate of $12 \%$ [5]. These have corresponded to median progression-free survival rates of 10 months [4] and 2.6 months [5] for combination and single-agent therapy respectively.

For patients with early stage disease, surgical resection with sentinel lymph node evaluation or bilateral inguinofemoral lymph node dissection is the standard. Determination of surgical margins and lymph node involvement informs the decision for re-excision or adjuvant external beam radiation (RT). Patients with lymph node involvement could also be considered for RT with or without concurrent chemotherapy with cisplatin, cisplatin plus fluorouracil (5-FU) or 5-FU plus mitomycin-C [6].

For patients with regional lymph node metastasis, extra nodal extension of the tumor, or fixed/ulcerated nodal metastasis (FIGO III/IVA), upfront surgical debulking results in significant morbidity and mortality $[7,8]$. Single-agent platinum therapy is inefficacious for this disease, as illustrated by the absence of any clinical responses to cisplatin monotherapy [9]. Chemoradiation has been shown to offer significant improvements in response rate, relapse-free survival and overall survival over RT in patients with locally advanced disease [10]. As such, upfront chemoradiation has been the generally employed standard. GOG 205 evaluated the clinical response rates in patients with locally advanced squamous cell vulvar carcinoma treated with cisplatinsensitized radiation therapy [11]. They reported a $64 \%$ response rate, allowing for many of these patients to subsequently undergo consolidative surgery. The addition of consolidative surgery after chemoradiation does not confer any significant increase in overall survival over chemoradiation alone [12]. Although the addition of chemotherapy to radiation has been shown to improve survival time to up to 44 months in patients with nodepositive disease [13], there is room to increase recurrence free survival in this patient population.

\section{Rationale for immunotherapy and platinum-sensitized radiotherapy}

Platinum agents such as cisplatin, carboplatin and oxaliplatin have long been hypothesized to promote immunogenic modulation outside of their DNA-platinum adduct formation and inhibition of DNA replication $[14,15]$. Lesterhuis et al. showed that human dendritic cells exposed to cisplatin induced significantly increased T-cell proliferation [16]. Furthermore, T-cells activated by platinum-sensitized DC's secreted increased levels of IFN- $\gamma$ and IL-2 [16]. Importantly, the authors found that this effect was in-part due to down-regulation of PD-L1 and PD-L2 on DCs. Finally, they found that cisplatinmediated STAT6 dephosphorylation led to PD-L1/2 downregulation [16]. In other reports, upregulation of mannose-6-phosphate (M6P) by cisplatin sensitized tumor cells to granzyme-B killing, and cytotoxic T-cellmedicated killing [17]. Cisplatin, has also been shown to induce immunogenic cell death via modulation of STAT signaling [18].

Ionizing radiation (RT) therapy induces IFN- $\gamma$, type I IFN production and PD-1/PD-L1 expression on tumor cells [19]. When combined with cisplatin, the immunogenic effect of RT is further potentiated via calreticulin exposure, release of ATP, induction of programmed death receptor 1-ligand (PD-L1) and high-mobility protein box-1 (HMGB-1) [20, 21]. Finally, there are several preclinical and clinical studies supporting the rational combination of immune checkpoint inhibitors with RT. For instance, combination therapy resulted in increased tumor infiltration of cytotoxic CD8 T-cells, decreased regulatory $\mathrm{T}$-cells and myeloid derived suppressor cells in melanoma and glioma tumor models [22, 23]. Furthermore, combination therapy enhanced antigen-cross presentation via upregulation of major histocompatibility complex (MHC) class I (MHC-I) [24] potentially priming the immune system for checkpoint blockade.

Lastly, immunotherapy maintenance after immunogenic interventions such as local ablative therapy or radiation therapy has been shown to be beneficial. For 
instance, patients with non-small cell lung (NSCLC) cancer treated with maintenance pembrolizumab after ablative therapy on a phase 2 study demonstrated statistically significant improvement in progression free survival (PFS) compared to historic controls [25]. In another report by Bersanelli et al. [26], patients who revived concurrent immunotherapy with radiotherapy had a longer PFS compared to patients who received radiation alone. A randomized placebo-controlled phase 3 trial evaluated the role of maintenance durvalumab in patients with NSCLC who had received chemoradiotherapy and found significant improvements in overall response rate, 12-month PFS, 18-month PFS and duration of response [27]. Notably, these combinations were well-tolerated.

\section{Methods}

\section{Aim and study design}

The purpose of this study is to capitalize on the immunogenic properties of concurrent cisplatin/radiation via addition of pembrolizumab. Addition of pembrolizumab to cisplatin-sensitized radiation (Cis-RT) therapy is hypothesized to increase overall response rate, and recurrence free survival via increased cytotoxic $\mathrm{T}$-cell engagement and activation. Based on this hypothesis, immunotherapy would need to be given concurrently with Cis-RT. Unlike CTLA-4 inhibitors, pembrolizumab has not been shown to have a dose-dependent relationship to toxicity with the currently utilized dose of $200 \mathrm{mg} / \mathrm{m}^{2}$ every 3 weeks. Due to this, a phase 1 trial with varying doses of pembrolizumab would not be instructive regarding toxicity and would likely impact potential efficacy. Also, due to the long half-life and frequency of administration, to test the hypothesis of synergy outlined in this study, pembrolizumab has to be present when CisRT is given. This makes a phase 2 trial design with pembrolizumab lead-in less ideal. In light of this, we decided to pursue a single-arm phase 2 clinical trial of concurrent Cis-RT and pembrolizumab. Finally, published and emerging toxicity data from immunotherapy plus chemoradiation combinations in other solid tumors have been reassuring, as described in the discussion section. The first three (3) patients with locally advanced or unresectable disease will receive cycle 1 of pembrolizumab followed by a break and resumption of pembrolizumab at cycle 4 and as part of a safety cohort. These patients will receive a total of 10 cycles of pembrolizumab (Fig. 1). All other patients, including the fourth patient with locally advanced/unresectable disease, will receive weekly cisplatin and pembrolizumab every 3 weeks, concurrently with daily radiation therapy (Fig. 2). Following completion of Cis-RT, patients will continue pembrolizumab maintenance for a total of 10 cycles in the safety cohort or 12 cycles for all other participants. Archived tissue will be used for MSI, PD-L1 and TIL stratification post hoc. Imaging will be performed at baseline and every 3 cycles (21-day cycles) as per standard-of-care. Laboratory analysis will occur on the first day of each cycle. We will collect and evaluate HPV status, PD-L1, and MSI status prior to therapy, but this will not be used for eligibility. Radiographic imaging will be performed every 9 weeks

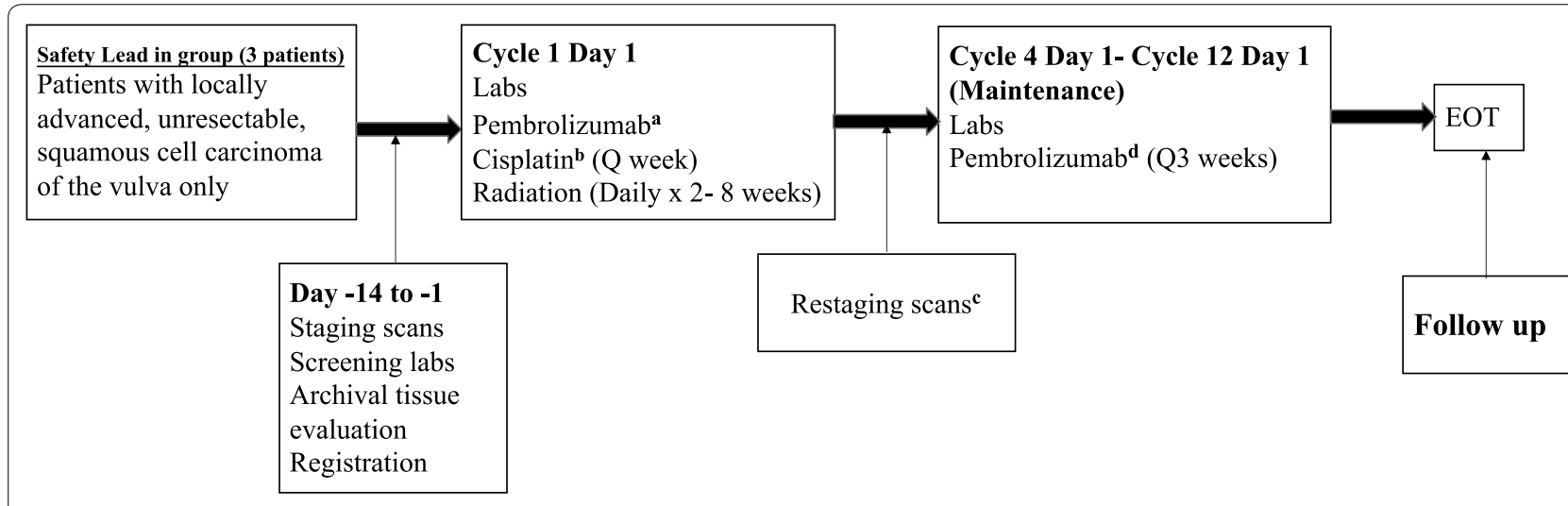

a: Pembrolizumab on cycle 1 day 1 and then resumed at cycle 4 day 1 . Each cycle is 21 days counted from the day of pembrolizumab administration

b: Cisplatin is given weekly on day 1 ( +3 days), during radiation therapy only

c: Restaging scans are performed after every $9(+/-1)$ weeks from C1D1 through week 36 or end of treatment (EOT). Then scans will be performed every 18 weeks for the next 36 weeks

d: Pembrolizumab continues Q3 weeks for 10 cycles in total

Fig. 1 Treatment schema for the first three patients with unresectable disease as part of the safety cohort 


\begin{tabular}{|c|c|c|c|c|}
\hline $\begin{array}{l}\text { Patients with locally } \\
\text { advanced, unresectable, } \\
\text { metastatic, or recurrent } \\
\text { squamous cell carcinoma } \\
\text { of the vulva }\end{array}$ & & $\begin{array}{l}\text { Cycle } 1 \text { Day } 1 \\
\text { Labs } \\
\text { Pembrolizumab (Q3 weeks) } \\
\text { Cisplatin }^{\mathbf{b}} \text { (Q week) } \\
\text { Radiation (Daily x 2- } 8 \text { weeks) }\end{array}$ & $\begin{array}{l}\text { Cycle } 4 \text { Day 1- Cycle } 12 \text { Day } 1 \\
\text { (Maintenance) } \\
\text { Labs } \\
\text { Pembrolizumab }{ }^{\mathrm{d}} \text { (Q3 weeks) }\end{array}$ & EOT \\
\hline & $\begin{array}{l}\text { Day -14 to -1 } \\
\text { Staging scans } \\
\text { Screening labs } \\
\text { Archival tissue } \\
\text { evaluation } \\
\text { Registration }\end{array}$ & Res & $a n s^{c}$ & Follow up \\
\hline $\begin{array}{l}\text { a: Pembrolizumab is } \\
\text { pembrolizumab admi } \\
\text { b: Cisplatin is given } \\
\text { c: Restaging scans ar } \\
\text { treatment (EOT). The } \\
\text { d: Pembrolizumab co }\end{array}$ & $\begin{array}{l}\text { s given Q3 wee } \\
\text { hinistration } \\
\text { weekly on day } \\
\text { re performed a } \\
\text { hen scans will b } \\
\text { continues Q3 w }\end{array}$ & $\begin{array}{l}\text { ks (every } 21 \text { days). Each cycle is } \\
1(+3 \text { days), during radiation the } \\
\text { fter every } 9(+/-1) \text { weeks from } C \\
\text { ee performed every } 18 \text { weeks for } \\
\text { eeks for } 12 \text { cycles in total }\end{array}$ & $\begin{array}{l}\text { counted from the day of } \\
\text { y } \\
36 \text { weeks week } 36 \text { or end of }\end{array}$ & \\
\hline
\end{tabular}

and overall response rate will be determined by RECIST 1.1 criteria.

\section{Patient characteristics}

For this study, women $\geq 18$ years with histologically or cytologically confirmed unresectable, incompletely resected, recurrent, or metastatic squamous cell carcinoma of the vulva will be eligible. Patients with unresectable disease defined as having $\mathrm{T} 2$ or $\mathrm{T} 3$ primary tumors (N0-3, M0) not amenable to surgical resection by standard radical vulvectomy will be eligible. Patients who have received prior chemotherapy, immunotherapy or radiation therapy will also be eligible. For patients who have received prior radiation therapy, re-irradiation to a previously treated site will not be permitted (Table 1).

\section{Study procedures}

After review and signing of written informed consent and trial registration, participants will be screened for eligibility and participation in the clinical trial. Pembrolizumab may be administered up to 1 day before or after the scheduled day 1 of each cycle due to administrative reasons. Cisplatin should be given on day 1 of every cycle. At some centers, cisplatin is administered on day 3 for administrative reasons and this will be allowable on study after notification of the PI during enrollment. The first 3 patients receiving definitive chemoradiation will receive pembrolizumab on cycle 1 only and then resume on cycle 4 after completion of chemoradiation (Fig. 1). This is for the first 3 patients with locally advanced, unresectable disease only. All other patients will be treated with concurrent pembrolizumab-cisplatin-radiation throughout (Fig. 2). For logistical reasons, patients are allowed to receive standard-of-care cisplatin-radiation locally. For these patients, radiation treatment plans are to be submitted to the radiation oncologist PI. These patients must also be available for $\mathrm{AE}$ assessments in person on day 1 of every cycle (pembrolizumab administration days) and weekly AE assessments by telephone. All trial treatments will be administered on an outpatient basis. Radiation therapy can be administered before or after immunotherapy or chemotherapy.

Pembrolizumab $200 \mathrm{mg}$ will be administered as a 30-min IV infusion every 3 weeks. Pembrolizumab will be administered first, prior to administration of cisplatin. Pembrolizumab will be continued for a total of 12 cycles, except for patients in the safety cohort who will receive a total of 10 cycles. After premedication, cisplatin $40 \mathrm{mg} / \mathrm{m}^{2}$ will be administered as a 30-60 min IV infusion (depending on institutional guidelines) weekly during radiation therapy. Cisplatin should be administered at the beginning of the week during radiation therapy. To allow for variation of administration of cisplatin at various clinical sites, cisplatin administration up to day +3 will be allowed. This request will be noted prior to patient enrollment. Cisplatin will only be administered during radiation therapy, minimum of 2 weeks, maximum of 8 weeks.

For patients with locally advanced or unresectable disease, external beam radiotherapy will be directed to 
Table 1 Inclusion and exclusion criteria

\author{
Inclusion criteria \\ Histologically or cytologically confirmed unresectable, incompletely \\ resected, recurrent, or metastatic squamous cell carcinoma of the vulva. \\ Patients with unresectable disease are defined as T2 or T3 primary \\ tumors (N0-3, M0) not amenable to surgical resection by standard radi- \\ cal vulvectomy \\ Participants must have measurable disease based on RECIST 1.1. Lesions \\ situated in a previously irradiated area are considered measurable if \\ progression has been demonstrated in such lesions \\ Documented Microsatellite stability status by routine methods including \\ MMR IHC, MSI PCR or MSI by Next Generation Sequencing (NGS) \\ Participants with no prior therapy are eligible and patients with recurrent \\ disease must not have had more than two lines of cytotoxic therapy \\ ECOG performance status of 0 or 1 \\ Absolute neutrophil count (ANC): $\geq 1500 / \mu \mathrm{L}$ \\ Platelets: $\geq 100,000 / \mu \mathrm{L}$ \\ Hemoglobin: $\geq 9.0 \mathrm{~g} / \mathrm{dL}$ or $\geq 5.6 \mathrm{mmol} / \mathrm{L}$ \\ Creatinine: $\leq 1.5 \times$ ULN \\ AST (SGOT) and ALT (SGPT): $\leq 2.5 \times$ ULN \\ Total bilirubin: $\leq 1.5 \times$ ULN \\ ULM upper limit of normal
}

the vulva and regional lymph nodes as determined by primary tumor location, including but not limited to inguinofemoral, external and internal iliac nodal regions. Treatment will adhere as closely as possible to standard of care. Any deviations will be submitted for approval to the radiation oncology PI prior to initiation of therapy. Patients with localized recurrent and/or metastatic disease with vulvar or inguinal and/or pelvic involvement and no prior history of radiation therapy are also candidates for tumor-directed radiotherapy to the site of recurrence. Patients with recurrent metastatic disease and a history of prior radiation can be considered if the target lesions are outside the prior radiation field, symptomatic, or if in the opinion of the investigator the patient can safely receive and benefit from additional radiation. CT simulation is required to define the gross tumor volume (GTV), clinical target volume (CTV), and planning target volume (PTV). The CT scan must be acquired in the same position and immobilization device as for treatment. The vulvar GTV and the groin GTV when inguinal nodes are unresectable will receive 68-70 Gy in 32-38 fractions of 1.8-2.2 Gy per fraction, provided organs at risk (OAR) metrics are met. Dose painting is permitted. Treatment will be delivered once daily, 5 days per week for the treatment duration. Breaks from treatment should be minimized and reasons for breaks must be documented. The groin PTV when high-risk positive nodal features are present after lymph-node dissection will receive $60 \mathrm{~Gy}$ in 30 fractions. Uninvolved pelvic or inguinal nodes should receive 45-50.4 Gy. Patients with

\section{Exclusion criteria}

Patients who in the opinion of the investigator cannot safely receive a minimum of $30 \mathrm{~Gy}$ in 10 fractions are not eligible for the trial

Participants who have received prior systemic anti-cancer therapy including investigational agents within 4 weeks prior to first dose of study treatment

Participants who have received a live vaccine within 30 days prior to the first dose of study drug

Participants with a history of gastrointestinal or colovesicular fistulae

Has active autoimmune disease that has required systemic treatment in the past 2 years (i.e. with use of disease modifying agents, corticosteroids or immunosuppressive drugs)

Has known active CNS metastases and/or carcinomatous meningitis unresectable/unresected pelvic nodes will receive radiation to a dose of $64 \mathrm{~Gy}$, as OARs allow. For patients with a prior history of radiation, no overlap of previously treated sites will be permitted. Thoracic metastasis will not to be irradiated. Treatment of bone metastases are allowed. Patients who in the opinion of the investigator cannot safely receive a minimum of 30 Gy in 10 fractions for metastatic disease are not eligible for the trial.

\section{Expected toxicities and management}

Toxicity will be graded using CTCAE version 5.0, and treatment criteria will be evaluated prior to each cycle (Table 2). Participants will be followed for up to 3 years or until death, whichever occurs first. Participants removed from protocol therapy for unacceptable adverse event(s) will be followed until resolution or stabilization of the adverse event. Survival status will be checked every 6 months during that time. Participants removed from study for unacceptable adverse events will be followed until resolution or stabilization of the adverse event. Expected toxicities from cisplatin include nausea, diarrhea, nephrotoxicity, tinnitus, ototoxicity, neutropenia, and thrombocytopenia. In the cases of grade 3 or 4 nausea, cisplatin would be held, and dose reduced (Table 3 ). Commonly seen adverse events seen with pembrolizumab include immunemediated pneumonitis, colitis, hepatitis, hypophysitis, hypo/hyperthyroidism, thyroiditis, type 1 diabetes mellitus, nephritis and rashes including; Stephens-Jonson syndrome (SJS) and Toxic epidermal necrolysis 
Table 2 Treatment criteria for the first cycle and subsequent cycles

\begin{tabular}{|c|c|}
\hline Cycle 1 criteria & Subsequent cycles \\
\hline Absolute neutrophil count $\geq 1500 / \mathrm{mcL}$ & Absolute neutrophil count $\geq 500 / \mathrm{mcL}$ \\
\hline Platelets $\geq 100,000 / \mathrm{mcL}$ & Platelets $\geq 50,000 / \mathrm{mcL}$ \\
\hline Hemoglobin $\geq 9 \mathrm{~g} / \mathrm{dL}$ & AST $(\mathrm{SGOT}) / \mathrm{ALT}(\mathrm{SGPT}) \leq 2.5 \times$ institutional upper limit of normal \\
\hline Total bilirubin $\leq 1.5 \times$ institutional upper limit of normal & Creatinine $\leq 1.5 \times$ ULN or creatinine clearance $\geq 50 \mathrm{~mL} / \mathrm{min}$ for subjects \\
\hline AST (SGOT)/ALT $(S G P T) \leq 2.5 \times$ institutional upper limit of normal & with creatinine levels above institutional ULN \\
\hline $\begin{array}{l}\text { Creatinine } \leq 1.5 \times \text { ULN or creatinine clearance } \geq 50 \mathrm{~mL} / \mathrm{min} \text { for subjects with } \\
\text { creatinine levels above institutional ULN }\end{array}$ & $\begin{array}{l}\text { All toxicities of previous cycles must have resolved to } \leq \text { grade } 2 \\
\text { ECOG performance of } 0 \text { to } 2\end{array}$ \\
\hline $\begin{array}{l}\text { All toxicities of previous therapy (aside from alopecia) must have resolved } \\
\text { to } \leq \text { grade } 1\end{array}$ & No evidence of life-threatening medical problems \\
\hline \multicolumn{2}{|l|}{ ECOG performance of 0 or 1} \\
\hline No evidence of life-threatening medical problems & \\
\hline
\end{tabular}

ULM upper limit of normal

Table 3 Toxicity and treatment holds

\begin{tabular}{|c|c|c|}
\hline & Management/next dose for cisplatin & $\begin{array}{l}\text { Management/ } \\
\text { next dose } \\
\text { for pembrolizumab }\end{array}$ \\
\hline \multicolumn{3}{|c|}{ Nausea/vomiting } \\
\hline$\leq$ Grade 1 & No change in dose & No change in dose \\
\hline Grade 2 & No change in dose & No change in dose \\
\hline Grade 3 & Hold ${ }^{\mathrm{a}}$ until < Grade 2. Resume at one dose level lower, if indicated. & No change in dose $e^{b, c}$ \\
\hline Grade 4 & Hold ${ }^{\mathrm{a}}$ until < Grade 2. Resume at two dose levels lower. & No change in dose $e^{b, c}$ \\
\hline \multicolumn{3}{|l|}{ Diarrhea } \\
\hline$\leq$ Grade 1 & No change in dose & No change in dose \\
\hline Grade 2 & No change in dose & No change in dose \\
\hline Grade 3 & Hold ${ }^{\text {a }}$ until $<$ Grade 2. Resume at one dose level lower, if indicated & No change in dose $e^{b, c}$ \\
\hline Grade 4 & Hold ${ }^{\mathrm{a}}$ until < Grade 2. Resume at two dose levels lower & No change in dose $e^{b, c}$ \\
\hline \multicolumn{3}{|l|}{ Neutropenia } \\
\hline$\leq$ Grade 1 & No change in dose & No change in dose \\
\hline Grade 2 & No change in dose & No change in dose \\
\hline Grade 3 & No change in dose & No change in dose \\
\hline Grade 4 & Hold ${ }^{d}$ until < Grade 3. Resume at current dose. & Hold until $\leq$ Grade 2 \\
\hline \multicolumn{3}{|c|}{ Thrombocytopenia } \\
\hline$\leq$ Grade 1 & No change in dose & No change in dose \\
\hline Grade 2 & No change in dose & No change in dose \\
\hline Grade 3 & Hold ${ }^{d}$ until $<$ Grade 2. Resume at same dose level & No change in dose \\
\hline Grade 4 & Hold $^{d}$ until $<$ Grade 2. Resume at same dose level & Hold until $\leq$ Grade 2 \\
\hline
\end{tabular}

(TEN). Similarly, management outlines for neutropenia and thrombocytopenia are also shown in Table 3. In the event of suspected or confirmed immune-related adverse events (irAEs) attributed to pembrolizumab, guidelines for management are detailed in Table 4 . Adverse events from radiation therapy include rashes, wounds, and other cutaneous manifestations, dysuria, and diarrhea.
Severe diarrhea could be from cisplatin, radiation, or pembrolizumab, and precise attribution might be challenging in some cases. To prevent patients receiving definitive therapy from unnecessary treatment breaks and delays due to suspected superimposed immunerelated colitis, the first three patients with locally advanced/unresectable disease will get pembrolizumab on cycle 1 day 1 and omit further pembrolizumab until 


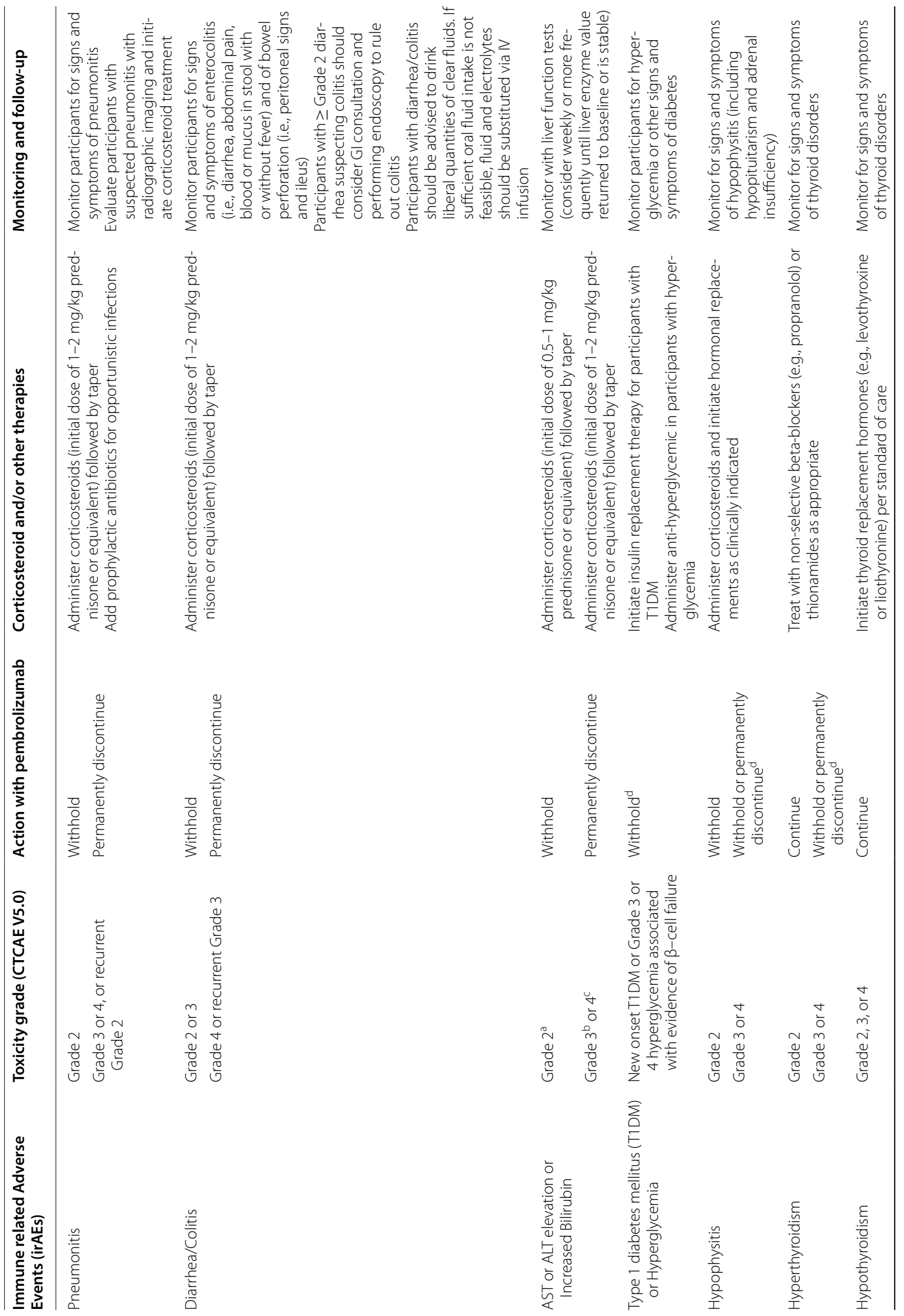




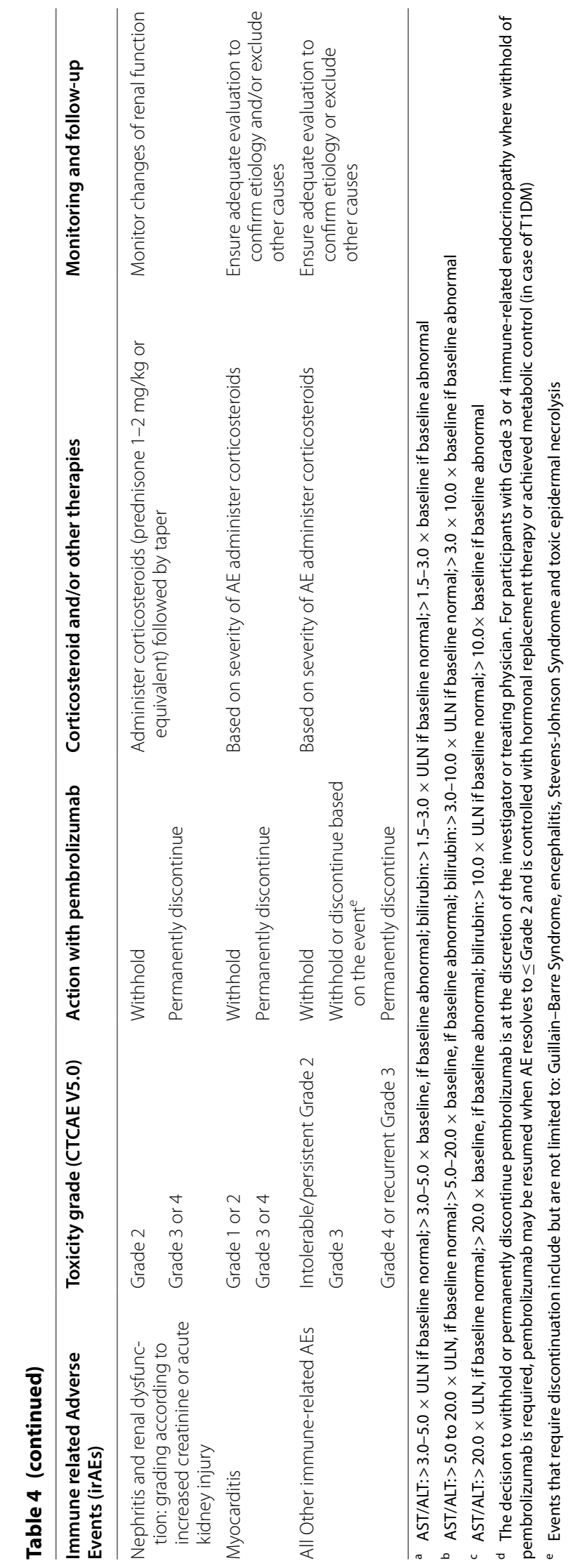


cycle 4 day 1 . If no unexpected toxicities are seen, all remaining patients in this category will receive pembrolizumab uninterrupted every 3 weeks.

\section{Translational studies}

Multiplex IHC will be performed on all archival samples for determination of; (i) CD3+, CD8+TILs, CD8+/CD4+FOXP3+TIL ratio, CD137+CD8+TILs, CD137+CD8+/CD4+FOXP3+TIL ratio, peritumoral lymphocytes and correlation with response; (ii) expression of immune checkpoints including TIM-3, LAG3, CTLA-4, PD-L2, PD-L1, PD-1, and correlation with response; (iii) targeted next generation $\mathrm{T}$-cell receptor sequencing (Adaptive biotechnologies) to determine T-cell clonality and correlate with response. Multiparameter flow cytometry will be used to determine the effect of treatment on circulating CD8+, CD4+FOXP3+Tcells. Cytokine analysis will also be performed to measure IFN- $\alpha$, IFN- $\beta$, TNF- $\alpha$, IL-6, IL-10, IL-12, and IL-2 levels and correlated with response. This will allow for comprehensive baseline profiling that will be correlated with treatment response. This approach has been validated as a means to determine the potential predictive value of immune cell phenotype and spatial distribution relative to the tumor [28]. This might allow for a better understanding of patients who might benefit from this form of combined therapy. We will collect HPV status, and MSI status at baseline. Identification of microsatellite instability resulting from defects in the mismatch repair pathway and specifically within MLH1, MSH2, MSH6 and PMS2 have been suggested to portend a significantly improved response to checkpoint blockade [29]. This information will be correlated with treatment response. One of the key mechanisms via which cisplatin-sensitized radiation therapy mediates its immunogenic effect is via calreticulin exposure, release of ATP, induction of programmed death receptor 1-ligand (PD-L1) and high-mobility protein box-1 (HMGB-1) [20, 21]. As such, we will measure baseline serum HMGB1 and at cycle 1, day 1 (D1), D5, D8, D12, D15, D19, D22 and D26 while on combination therapy via commercially available ELISA assays.

\section{Statistical considerations}

The target enrolment is 24 patients. The primary endpoint for this study is overall response rate (ORR). This sample size calculation is based on an ORR of $\geq 60 \%$. For recurrent metastatic disease, there are is no standard of care. The ORR estimation is informed by data showing an ORR of $40 \%$ in patients with advanced or recurrent metastatic vulvar cancer treated with platinum-based combination therapy [4]. Single-agent chemotherapy has an ORR of about $12 \%$ [5]. Patients with primary disease who are not candidates for upfront surgery have a reported response rates from 55\% [30] to 64\% [11]. Only patients who undergo treatment on protocol will be eligible for analysis. Patients who sign consent and do not undergo any treatment will be ineligible for evaluation. With $\mathrm{n}=24$, the power to reject Ho: ORR $\leq 30 \%$ in favor of $\mathrm{H} 1:$ ORR $\geq 60 \%$ will be $89 \%$ at a target significance level of 0.05 for a one-sided exact Binomial test (12 or more responses are required to reject Ho in favor of $\mathrm{H} 1$ ). Sixmonth Recurrence Free Survival (RFS-6) is the secondary endpoint. Exploratory biomarkers include; Predictive values of baseline dMMR/MSI-status and PD-L1; Antitumor inflammatory responses of cGas-STING- pathway, systemic inflammatory cytokines, and circulating T-cell receptor repertoire.

\section{Analysis strategy}

Exact Binomial test and 95\% CI for ORR (primary endpoint). For the secondary objectives of RFS-6, a point estimate with exact 95\% CI and Kaplan-Meier estimate will be used for the RFS distribution. Operating characteristics of prediction (sensitivity, specificity, positive- and negative predictive values with $95 \% \mathrm{CI}$ ) for the baseline dMMR/MSI-status and PD-L1, and descriptive analysis will be used for anti-tumor inflammatory cytokines and HMGB-1 levels.

\section{Discussion}

There are numerous clinical trials that support safety of the combination of chemotherapy with immune checkpoint blockade [31-33]. In addition, there is prospective safety data for combining chemoradiation with immune checkpoint blockade. Phase 2 and 3 clinical trials in NSCLC evaluating concurrent platinum-based chemotherapy with RT and PD-L1 blockade reported an incidence of about $30 \%$ in adverse related events [27, 34]. This is not significantly higher than what would be expected for chemoradiation treatment alone. A phase 1 trial by Tang et al. evaluated the combination of hypofractionated radiotherapy; 50 Gy in 4 fractions or 60 Gy in 10 fractions, with ipilimumab in patients with metastatic solid tumor malignancies [35]. In this study, 34\% of patients developed grade 3 toxicity with colitis being the most common adverse event. Of note, $23 \%$ of the patients treated on this trial derived benefit via abscopal effects [35]. Another phase I trial combined pembrolizumab with various courses of radiation therapy; $30 \mathrm{~Gy}$ in 3 fractions for osseous metastasis, $50 \mathrm{~Gy}$ in 5 fractions for central lung tumors and 45 Gy in 3 fractions for other sites [36]. Only $6 \%$ of the 62 participants in this trial experienced grade 3 toxicities. The authors also reported abscopal effects in $13.2 \%$ of these patients [36]. NRG-GY017 is an ongoing phase I clinical trial evaluating an anti-PD-L1 inhibitor, atezolizumab, in 
patients with node positive Stage IB2, II, IIIB, or IVA cervical cancer. Similar to this trial, both arms of the study include the administration of immune checkpoint blockade concurrently with cisplatin-sensitized radiation therapy at doses similarly used for GYN malignancies, and there have been no adverse safety signals reported (NCT 03738228). As a safety precaution, the first three patients with locally advanced unresectable disease treated on this trial with receive pembrolizumab on cycle 1 and then resume on cycle 4 and on (Fig. 1).

Several preclinical studies show that combination cisplatin and radiation therapy increases the immunogenetic effect of each treatment modality in ways that can be further enhanced by PD-1/PD-L1 blockade. By adding pembrolizumab early during the delivery of Cis$\mathrm{RT}$, the opportunity for antigen capture and presentation, DC priming and T-cell activation is potentially increased. The pragmatic design of this trial also allows for collection of translational correlatives to evaluate if a systemic anti-tumor inflammatory response is being generated by this triplet combination.

In a rare disease with limited options in the recurrent setting, addition of immune checkpoint blockade to cisplatin-sensitized radiation could provide durable disease control by engaging the immune system.

\begin{abstract}
Abbreviations
PD-L1: Programmed death receptor 1-ligand; PD-L2: Programmed death receptor 2-ligand; HPV: Human papillomavirus; TIL: Tumor infiltrating Lymphocytes; MSI: Microsatellite instability; dMMR: Deficient mismatch repair; IL-2: Interleukin 2; DC: Dendritic cell; M6P: Mannose-6-phosphate; RT: Radiation therapy; PFS: Progression-free survival; PI: Principal investigator; AE: Adverse event; OS: Overall survival; NSCLC: Non small cell lung cancer; IFN- $\gamma$ : Interferon gamma; HMGB-1: High-mobility protein box-1; MHC: Major histocompatibility complex; AE: Adverse events; GTV: Gross tumor volume; CTV: Clinical tumor volume; PTV: Planning tumor volume; OAR: Organs at risk; CTCAE: Common terminology criteria for adverse events; IHC: Immunohistochemistry; ELISA: Enzyme-linked immunosorbent assay; ORR: Overall response rate; RFS: Relapse free survival.
\end{abstract}

\section{Acknowledgements}

We would like to thank Jennifer Filipi NP, Tina Colella RN, Kellie Redmond and Sara Bouberhan MD, for review of the protocol design and critical feedback.

\section{Authors' contributions}

OY conceived of the study, designed the trial, wrote the protocol and is the principal investigator. AR designed the trial, wrote the protocol and is the radiation oncology PI. HL designed the trial and wrote the protocol. DS designed the trial and wrote the protocol. OY, ALR, HL and DS wrote the manuscript. All authors read and approved the final manuscript.

\section{Funding}

Merck is funding the study as part of an investigator initiated study. Merck did not contribute to the study design, or translational design and analysis. Merck provided input on adverse event reporting for pembrolizumab. National Institutes of Health, National Cancer Institute grant P01 CA190174 (D.S and O.Y). Executive Committee on Research Physician Scientist Development Award (O.Y).

\section{Availability of data and materials}

At the completion of the trial, de-identified data will be available upon request.

\section{Ethics approval and consent to participate}

This study has been approved by the Institutional Review Board (IRB). The approval is granted by The Office for Human Research Studies (OHRS) at the Dana Farber Harvard Cancer Center.

\section{Consent for publication}

Not applicable.

\section{Competing interests}

O.Y has no competing interests.

\section{Author details}

${ }^{1}$ Division of Hematology-Oncology, Department of Medicine, Harvard Medical School, Massachusetts General Hospital, Boston, USA. ${ }^{2}$ Department of Radiation Oncology, Harvard Medical School, Massachusetts General Hospital, Boston, USA. ${ }^{3}$ Biostatistics Center, Harvard Medical School, Massachusetts General Hospital, Boston, USA.

Received: 23 June 2020 Accepted: 8 September 2020

Published online: 14 September 2020

\section{References}

1. Siegel RL, Miller KD, Jemal A. Cancer statistics, 2018. CA Cancer J Clin. 2018;68(1):7-30.

2. Francis JA, Eiriksson L, Dean E, Sebastianelli A, Bahoric B, Salvador S. No. 370-Management of squamous cell cancer of the vulva. J Obstet Gynaecol Can. 2019;41(1):89-101.

3. SEER Cancer Statistics Factsheets: Vulvar Cancer. Bethesda, MD: National Cancer Institute. http://seer.cancer.gov/statfacts/html/vulva.html. Accessed 8 Feb 2019.

4. Cormio G, Loizzi V, Gissi F, et al. Cisplatin and vinorelbine chemotherapy in recurrent vulvar carcinoma. Oncology. 2009;77(5):281-4.

5. Witteveen PO, van der Velden J, Vergote I, et al. Phase II study on paclitaxel in patients with recurrent, metastatic or locally advanced vulvar cancer not amenable to surgery or radiotherapy: a study of the EORTCGCG (European Organisation for Research and Treatment of CancerGynaecological Cancer Group). Ann Oncol. 2009:20(9):1511-6.

6. Horowitz NS, Olawaiye AB, Borger DR, et al. Phase II trial of erlotinib in women with squamous cell carcinoma of the vulva. Gynecol Oncol. 2012;127(1):141-6

7. Forner DM, Lampe B. Exenteration in the treatment of Stage III/IV vulvar cancer. Gynecol Oncol. 2012;124(1):87-91.

8. Miller B, Morris M, Levenback C, Burke TW, Gershenson DM. Pelvic exenteration for primary and recurrent vulvar cancer. Gynecol Oncol. 1995;58(2):202-5

9. Thigpen JT, Blessing JA, Homesley HD, Lewis GC Jr. Phase II trials of cisplatin and piperazinedione in advanced or recurrent squamous cell carcinoma of the vulva: a Gynecologic Oncology Group Study. Gynecol Oncol. 1986;23(3):358-63.

10. Han SC, Kim DH, Higgins SA, Carcangiu ML, Kacinski BM. Chemoradiation as primary or adjuvant treatment for locally advanced carcinoma of the vulva. Int J Radiat Oncol Biol Phys. 2000;47(5):1235-44.

11. Moore DH, Ali S, Koh WJ, et al. A phase II trial of radiation therapy and weekly cisplatin chemotherapy for the treatment of locally-advanced squamous cell carcinoma of the vulva: a gynecologic oncology group study. Gynecol Oncol. 2012;124(3):529-33.

12. van Doorn HC, Ansink A, Verhaar-Langereis M, Stalpers L. Neoadjuvant chemoradiation for advanced primary vulvar cancer. Cochrane Database Syst Rev. 2006. https://doi.org/10.1002/14651858.CD003752.pub2.

13. Gill BS, Bernard ME, Lin JF, et al. Impact of adjuvant chemotherapy with radiation for node-positive vulvar cancer: a National Cancer Data Base (NCDB) analysis. Gynecol Oncol. 2015;137(3):365-72.

14. Kelland $L$. The resurgence of platinum-based cancer chemotherapy. Nat Rev Cancer. 2007;7(8):573-84. 
15. Todd RC, Lippard SJ. Inhibition of transcription by platinum antitumor compounds. Metallomics. 2009;1(4):280-91.

16. Lesterhuis WJ, Punt CJ, Hato SV, et al. Platinum-based drugs disrupt STAT6-mediated suppression of immune responses against cancer in humans and mice. J Clin Invest. 2011;121(8):3100-8.

17. Ramakrishnan R, Assudani D, Nagaraj S, et al. Chemotherapy enhances tumor cell susceptibility to CTL-mediated killing during cancer immunotherapy in mice. J Clin Invest. 2010;120(4):1111-24.

18. Hato SV, de Vries IJ, Lesterhuis WJ. STATing the importance of immune modulation by platinum chemotherapeutics. Oncoimmunology. 2012;1(2):234-6.

19. Gong J, Le TQ, Massarelli E, Hendifar AE, Tuli R. Radiation therapy and PD-1/PD-L1 blockade: the clinical development of an evolving anticancer combination. J Immunother Cancer. 2018;6(1):46.

20. Martins I, Kepp O, Schlemmer F, et al. Restoration of the immunogenicity of cisplatin-induced cancer cell death by endoplasmic reticulum stress. Oncogene. 2011;30(10):1147-58.

21. Obeid M, Panaretakis T, Joza N et al. Calreticulin exposure is required for the immunogenicity of gamma-irradiation and UVC light-induced apoptosis. Cell Death Differ. 2007:14(10):1848-50.

22. Verbrugge I, Hagekyriakou J, Sharp LL, et al. Radiotherapy increases the permissiveness of established mammary tumors to rejection by immunomodulatory antibodies. Cancer Res. 2012;72(13):3163-74.

23. Zeng J, See AP, Phallen J, et al. Anti-PD-1 blockade and stereotactic radiation produce long-term survival in mice with intracranial gliomas. Int J Radiat Oncol Biol Phys. 2013;86(2):343-9.

24. Sharabi AB, Nirschl CJ, Kochel CM, et al. Stereotactic radiation therapy augments antigen-specific PD-1-mediated antitumor immune responses via cross-presentation of tumor antigen. Cancer Immunol Res. 2015;3(4):345-55.

25. Bauml JM, Mick R, Ciunci C, et al. Pembrolizumab after completion of locally ablative therapy for oligometastatic non-small cell lung cancer: a Phase 2 Trial. JAMA Oncol. 2019:5(9):1283-90

26. Bersanelli M, Lattanzi E, D'Abbiero N, et al. Palliative radiotherapy in advanced cancer patients treated with immune-checkpoint inhibitors: the PRACTICE study. Biomed Rep. 2020;12(2):59-67.

27. Antonia SJ, Villegas A, Daniel D, et al. Durvalumab after chemoradiotherapy in stage III non-small-cell lung cancer. N Engl J Med. 2017;377(20):1919-29.
28. Remark R, Merghoub T, Grabe N, et al. In-depth tissue profiling using multiplexed immunohistochemical consecutive staining on single slide. Sci Immunol. 2016;1(1):aaf6925.

29. Le DT, Uram JN, Wang H, et al. PD-1 blockade in tumors with mismatchrepair deficiency. N Engl J Med. 2015;372(26):2509-20.

30. Lee J, Kim SH, Kim G, et al. Treatment outcome in patients with vulvar cancer: comparison of concurrent radiotherapy to postoperative radiotherapy. Radiat Oncol J. 2012;30(1):20-6.

31. Barker CA, Postow MA, Kronenberg SA, Ma J, Yamada Y, Beal K, et al. Concurrent radiation therapy (RT), ipilimumab (Ipi) and/or nivolumab (nivo) on a phase 1 clinical trial [abstract]. Int J Radiat Oncol Biol Phys. 2015. https://doi.org/10.1016/j.ijrobp.2015.07.506.

32. Duffy AG, Kleiner DE, Alewine C, Figg WD, Steinberg SM, et al. A pilot study of immune checkpoint inhibition in combination with radiation therapy in patients with metastatic pancreatic cancer [abstract]. J Clin Oncol. 2017. https://doi.org/10.1200/jco.2017.35.4_suppl.316.

33. Levy A, Massard C, Soria JC, Deutsch E. Concurrent irradiation with the anti-programmed cell death ligand-1 immune checkpoint blocker durvalumab: single centre subset analysis from a phase 1/2 trial. Eur I Cancer. 2016:68:156-62.

34. Lin SH LY, Price J, Parker M, Gomez DR, Welsh JW, et al. DETERRED: PD-L1 blockade to evaluate the safety of lung cancer therapy using carboplatin, paclitaxel, and radiation combined with MPDL3280A (atezolizumab) [abstract]. J Clin Oncol 2017; 35:Abstr nr 3064.

35. Tang C, Welsh JW, de Groot P, et al. Ipilimumab with stereotactic ablative radiation therapy: Phase I results and immunologic correlates from peripheral T cells. Clin Cancer Res. 2017:23(6):1388-96.

36. Luke JJ, Lemons JM, Karrison TG, et al. Safety and clinical activity of pembrolizumab and multisite stereotactic body radiotherapy in patients with advanced solid tumors. J Clin Oncol. 2018;36(16):1611-8.

\section{Publisher's Note}

Springer Nature remains neutral with regard to jurisdictional claims in published maps and institutional affiliations.
Ready to submit your research? Choose BMC and benefit from:

- fast, convenient online submission

- thorough peer review by experienced researchers in your field

- rapid publication on acceptance

- support for research data, including large and complex data types

- gold Open Access which fosters wider collaboration and increased citations

- maximum visibility for your research: over $100 \mathrm{M}$ website views per year

At BMC, research is always in progress.

Learn more biomedcentral.com/submissions 